\title{
Biodisponibilidade de ferro do açaí (Euterpe oleracea Mart.) e da farinha de mandioca fortificada com ferro em ratos ${ }^{1,2}$
}

\author{
Iron bioavailability of the açaí (Euterpe \\ oleracea Mart.) and the iron-fortified manioc \\ flour in rats
}

Sirlene Duarte Alves TOAIARI ${ }^{3}$

Lucia Kiyoko Ozaki YUYAMA ${ }^{3}$

Jaime Paiva Lopes AGUIAR ${ }^{3}$

Risonilce Fernandes Silva SOUZA ${ }^{3}$

RE S U M O

\section{Objetivo}

Este estudo teve como objetivo avaliar a biodisponibilidade do ferro do açaí (Euterpe oleracea Mart.) e da farinha de mandioca fortificada com ferro, utilizando o método de depleção e repleção de hemoglobina em ratos.

\section{Métodos}

No período de depleção, durante a lactação, as ratas (Rattus novergicus) e seus filhotes receberam ração à base de caseína (American Institute of Nutrition - 93G), sem adição de ferro na mistura salina, sendo que os filhotes, quando desmamados, continuaram a receber a mesma ração por mais sete dias. Paralelamente, houve um grupo controle no qual ratas e filhotes receberam ração comercial. No período de repleção de catorze dias, os ratos machos $(n=40)$ foram distribuídos aleatoriamente em cinco grupos de oito ratos cada um, os quais receberam rações experimentais (açaí e farinha de mandioca fortificada com ferro) e controles (do açaí e American Institute of Nutrition 93). A concentração de hemoglobina foi determinada semanalmente e a biodisponibilidade de ferro das rações foi determinada por meio de cálculos do ferro ingerido e do ferro hemoglobínico dos ratos dos diferentes grupos.

\footnotetext{
1 Artigo elaborado a partir da dissertação de S.D.A TOAIARI, "Biodisponibilidade de ferro do açaí (Euterpe oleracea Mart.) e de alimentos fortificados: pão e farinha de mandioca (Manihot esculenta Crantz.). Estudo em ratos". Universidade do Amazonas; 2002.

2 Estudo financiado pelo PPG-7/FINEP - Proc. 64.99.0477.00, INPA- PPI: 1-3100 e CAPES,

3 Coordenação de Pesquisas em Ciências da Saúde, Instituto Nacional de Pesquisas da Amazônia. Av. André Araújo, 2936, Petrópolis, 69083-000, Manaus, AM, Brasil. Correspondência para/Correspondence to: L. K.O. YUYAMA. E-mail: <yuyama@inpa.gov.br>.
} 


\section{Resultados}

Ao final do período de depleção, a concentração de hemoglobina dos ratos, de $4,7 \pm 0,5 \mathrm{~g} / \mathrm{dL}$, demonstrou a efetividade do método utilizado. A maior biodisponibilidade de ferro foi constatada na farinha de mandioca fortificada $(44,6 \pm 3,6 \%)$, em contraste com a baixa biodisponibilidade do ferro do açaí $(12,1 \pm 5,5 \%)$.

\section{Conclusão}

O ferro presente no açaí não foi eficaz na recuperação da concentração de hemoglobina dos ratos. Portanto sugere-se cautela ao recomendar a utilização do açaí como fonte de ferro.

Termos de indexação: açaí, anemia, disponibilidade biológica, farinha, ferro, hemoglobina, mandioca, ratos.

\section{A B S T R A C T}

\section{Objective}

This study evaluates the bioavailability of iron derived from açaí (Euterpe oleracea Mart.) and from ironfortified manioc flour, using the hemoglobin iron-depletion-recovery method in rats.

\section{Methods}

A casein-based diet (American Institute of Nutrition - 93G), without added iron in the saline mixture, was fed to female Wistar rats during the 21-day lactation period; it was also fed to the pups, for 7 days after weaning (depletion period). Simultaneously, a control group of female rats and pups received a commercial feed. In the 14 days repletion period, male rats $(n=40)$ were randomly distributed in five groups and fed one of the diets: control (açaí control and American Institute of Nutrition - 93) or experimental (açaí and iron-fortified manioc flour). The hemoglobin concentration was determined weekly. The iron bioavailability was obtained through the heme-iron/iron intake ratios, calculated for each group.

\section{Results}

The mean hemoglobin concentration of $4.7 \pm 0.5 \mathrm{~g} / \mathrm{dL}$ at the end of the depletion period demonstrated the effectiveness of the iron-depletion method used in the study. It was observed that the greatest iron bioavailability was drawn from the fortified manioc flour $(44.6 \pm 3.6 \%)$, in contrast with the low iron bioavailability derived from açaí $(12.1 \pm 5.5 \%)$.

\section{Conclusion}

The iron present in the açaí was not effective in recovering the hemoglobin concentration in the rats. Hence, any recommendation for the use of açaí as a source of iron should be treated with caution.

Indexing terms: açaí, anemia, biological availability, flour, iron, hemoglobin, manihot, rats.

\section{N T R O D U Ç Ã O}

A anemia ferropriva é uma das enfermidades mais prevalentes em todo o mundo, particularmente nos países em desenvolvimento, sendo a deficiência de ferro a causa mais importante dessa carência'. Lactentes, pré-escolares, adolescentes e gestantes são os grupos de risco mais susceptíveis à anemia ferropriva, pelo aumento das necessidades de ferro decorrentes da rápida expansão dos tecidos e da massa de hemácias ${ }^{2}$. As principais conseqüências da anemia para a saúde desses grupos vulneráveis são alterações de crescimento, pele e mucosas, sistema digestório, função muscular, compor- tamento, imunidade e aumento da mortalidade e susceptibilidade às infecções em crianças acometidas por anemia grave ${ }^{3}$. O principal fator de risco para a instalação da anemia ferropriva é o dietético, por meio do consumo insuficiente de alimentos fontes e da baixa biodisponibilidade de ferro da dieta ${ }^{4}$.

Apesar do compromisso de priorizar o combate à anemia ferropriva assumido no Encontro Mundial de Cúpula pela Criança em Roma, em 1992, por 170 países, entre eles o Brasil, o problema ainda persiste. Tais constatações são evidenciadas, por exemplo, nos trabalhos de Monteiro et al. ${ }^{5}$, que demonstram a tendência 
secular do aumento da anemia ferropriva na infância em São Paulo, a maior cidade brasileira. Estudo realizado por Fisberg et al. ${ }^{6} \mathrm{em}$ dez capitais brasileiras constatou a prevalência média de $54,0 \%$ de anemia ferropriva em crianças menores de cinco anos. Não obstante, no Amazonas, estudos encontraram elevada prevalência de anemia ferropriva em lactentes, na ordem de $68,6 \%$, constituindo um importante problema de saúde pública ${ }^{7}$.

No Brasil, a redução da anemia ferropriva foi novamente priorizada entre as diretrizes da Política Nacional de Alimentação e Nutrição. Por meio da Resolução RDC n 344, de 13 de dezembro de 2002, da Agência Nacional de Vigilância Sanitária (ANVISA), tornou-se obrigatória a fortificação das farinhas de trigo e das farinhas de milho com ferro e ácido fólico. Segundo a ANVISA ${ }^{8}$, a partir da data de publicação dessa resolução, as empresas terão um prazo de dezoito meses para adequação de seus produtos. Esse programa segue uma tendência mundial: eleger a fortificação de alimentos como a estratégia mais indicada, especialmente em países em desenvolvimento.

Um dos componentes fundamentais de um programa de fortificação é a eleição dos alimentos que devem ser fortificados, sendo um dos critérios mais importantes o amplo consumo desse alimento pela população e seu baixo custo. Outro fator importante é a escolha do composto de ferro a ser utilizado: deve ser biodisponível, apresentar boa relação custo-benefício e não produzir alterações organolépticas no alimento, entre outras características ${ }^{9}$. Nesse contexto, o Grupo de Alimentos e Nutrição na Amazônia, do Instituto Nacional de Pesquisa da Amazônia, vem desenvolvendo estudos na linha de fortificação de alimentos objetivando a recuperação e prevenção dos processos carenciais, a exemplo da farinha de mandioca fortificada com ferro aminoácido quelato, e da utilização de alimentos com potencial nutricional, dentre eles o açaí (Euterpe oleracea Mart.).

Nativo da região amazônica, o açaí, fruto arredondado medindo de 1,0 a $1,5 \mathrm{~cm}$ de diâmetro, mesocarpo cerca de $1 \mathrm{~mm}$ de espessura, de cor atro-violácea quando maduro, é utilizado para a produção de uma bebida chamada "açaí" ou "vinho do açaí", muito apreciada e popularmente conhecida como fonte de ferro, sendo utilizada para combater a anemia ferropriva, sem, contudo, haver comprovação científica que respalde essa prática.

Avaliar cientificamente o potencial do açaí como fonte de ferro e a farinha de mandioca fortificada com ferro na recuperação da concentração de hemoglobina de ratos anêmicos foi a proposta do presente estudo.

\section{M É T O D O S}

\section{Açaí}

O açaí foi coletado na llha das Onças, Município de Belém, PA, e processado em uma fábrica local. Os frutos passaram por um processo de higienização em água potável e corrente, seguido da imersão em água com hipoclorito na proporção de 20ppm por litro, por 35 minutos em média. Da mesma forma, as máquinas foram pré-higienizadas com hipoclorito para posterior processamento do fruto e obtenção do suco de açaí; em seguida, o suco foi acondicionado em sacos plásticos de um litro, empacotado, congelado e transportado até Manaus. No Laboratório de Nutrição e Físico-Química de Alimentos da Coordenação de Pesquisas em Ciência da Saúde do Instituto Nacional de Pesquisas da Amazônia, o açaí foi descongelado e seco em estufa com circulação de ar forçada a $60^{\circ} \mathrm{C}$ até peso constante, para fins de determinação do teor de umidade. Amostras representativas foram retiradas aleatoriamente para a determinação da composição química.

\section{Farinha de mandioca}

A farinha de mandioca fortificada utilizada no experimento foi oriunda da Fábrica Caiari 
Agroindústria Ltda e o processamento consistiu das seguintes etapas: colheita da mandioca, lavagem, descascamento, puba (remolho), trituração, prensagem, homogeneização em cilindro elétrico, adição de ferro aminoácido quelato, homogeneização em misturador elétrico, torrefação, peneiramento e embalagem em sacos de polietileno e transporte até Manaus para fins de análises e utilização. O fortificante utilizado foi o glicinato de ferro (Ferrochel Amino Acid Chelate) da Albion Lab. Inc.

O delineamento experimental foi inteiramente casualizado e subdividido em dois períodos: depleção e repleção. No período de depleção (28 dias), foram utilizados dezenove ratas com seis filhotes machos cada uma (Rattus novergicus) da linhagem Wistar, provenientes do biotério do Instituto Nacional de Pesquisas da Amazônia. Durante a lactação (21 dias), dezessete ratas e seus respectivos filhotes receberam ração à base de caseína sem adição de ferro na mistura salina (Tabela 1), com o objetivo de induzir a deficiência de ferro, e duas ratas e seus respectivos filhotes receberam ração comercial. Após o desmame, os filhotes continuaram recebendo as mesmas rações por mais sete dias. Ao final do período, amostras de sangue foram coletadas pela cauda dos animais para determinação da concentração de hemoglobina e seleção para o período de repleção, com ponto de corte correspondente a $\mathrm{Hb}$ menor que $7 \mathrm{~g} / \mathrm{dL}$ para ratos anêmicos e $\mathrm{Hb}$ maior que $11 \mathrm{~g} / \mathrm{dL}$ para ratos normais ${ }^{10}$.

No período de repleção (14 dias), foram constituídos cinco grupos de oito ratos cada um $(n=40)$; o peso médio dos ratos anêmicos foi de $64,3 \pm 0,9 \mathrm{~g}$ e dos ratos normais, de $84,7 \pm 3,9 \mathrm{~g}$, distribuídos aleatoriamente segundo a concentração de hemoglobina: 1) grupo experimental - ração à base de açaí; 2) grupo experimental - ração à base de farinha de mandioca fortificada com ferro aminoácido quelato; 3) grupo-controle do açaí - ração controle do açaí; 4) grupo-controle 1 - ração à base de caseína; 5) grupo-controle 2 - ratos normais - ração comercial.

As rações oferecidas nesse período foram elaboradas de acordo com as recomendações de Reeves et al. ${ }^{11}$ (Tabela 1), contendo 35mg de $\mathrm{Fe} / \mathrm{kg}$ de ração e fornecidas "ad libitum" (exceto para o grupo 3, controle do açaí), juntamente com a água.

Durante o período de depleção de ferro, os animais foram mantidos em caixas de

Tabela 1. Composição percentual das rações do período de depleção e repleção.

\begin{tabular}{|c|c|c|c|c|c|}
\hline Ingredientes & AIN 1993 G (\%)* AIN & $1993 \mathrm{G}(\%)^{* *}$ & Farinha de mandioca $(\%)^{* *}$ & Açaí $(\%)^{* *}$ & Controle do açaí $(\%)^{* *}$ \\
\hline Caseína & 20,00 & 20,00 & 20,00 & 12,30 & 20,00 \\
\hline Sacarose & 10,00 & 10,00 & 10,00 & 2,85 & 10,00 \\
\hline Óleo de soja & 7,00 & 7,00 & 7,00 & - & 38,10 \\
\hline Fibra microcristalina & 5,00 & 5,00 & 5,00 & - & 10,00 \\
\hline Mistura salina & - & 3,50 & - & - & 3,50 \\
\hline Mistura salina sem Ferro & 3,50 & - & 3,50 & 3,50 & - \\
\hline Mistura vitamínica & 1,00 & 1,00 & 1,00 & 1,00 & 1,00 \\
\hline L-cistina & 0,30 & 0,30 & 0,30 & 0,18 & 0,30 \\
\hline Bitartarato de colina & 0,25 & 0,25 & 0,25 & 0,25 & 0,25 \\
\hline Pão fortificado com Ferro & - & - & - & - & - \\
\hline Açaí desidratado & - & - & - & 80,00 & - \\
\hline $\begin{array}{l}\text { Farinha de mandioca } \\
\text { fortificada com Ferro }\end{array}$ & - & - & 48,60 & - & - \\
\hline Amido de milho & 53,00 & 53,00 & 4,40 & - & 16,90 \\
\hline Total & 100,00 & 100,00 & 100,00 & 100,00 & 100,00 \\
\hline
\end{tabular}

* ração do período de depleção; ** rações do período de repleção; AIN 1993 G= American Institute of Nutrition ${ }^{11 .}$ 
polipropileno com tampas de aço inoxidável e, no período de repleção, em gaiolas individuais de aço inoxidável, com umidade e temperatura controladas e constantes, com um ciclo de luz de doze horas por dia. Os animais foram pesados semanalmente em balança semi-analítica e o controle do consumo de rações foi realizado diariamente.

Toda a vidraria utilizada foi desmineralizada com ácido nítrico a 30\% (per noite) e os recipientes à base de aço inoxidável imersos em solução de EDTA a $2 \%$ e enxaguados por no mínimo seis vezes com água desionizada.

As análises químicas em triplicata das rações foram realizadas para a determinação da composição centesimal segundo a Association of Official Analytical Chemists ${ }^{12}$; fibra alimentar, segundo Asp et al. ${ }^{13}$; tanino do açaí, segundo Reicher et al. ${ }^{14}$ e concentração de ferro, segundo o Instituto Adolfo Lutz ${ }^{15}$ e manual da Perkin-Elmer ${ }^{16}$. Após a digestão via úmida, em triplicata, a leitura direta em solução de amostras foi realizada em espectrofotômetro-Perkin Elmer modelo 1100 com lâmpada de catodo oco para ferro. Para o controle das análises utilizaram-se as recomendações de Cornelis $^{17}$, tendo como material de referência Peach leaves (NIST-SRM 1547).

Semanalmente foi coletado sangue pela cauda dos animais para a determinação da concentração de hemoglobina, utilizando-se o hemoglobinômetro portátil HemoCue ${ }^{\circledR}$. Esse método utiliza microcuvetas descartáveis com reagente de fórmula seca (desoxicolato de sódio, nitrito de sódio, azida de sódio e componentes não reativos) e um aparelho fotômetro. O desoxicolato de sódio hemolisa os eritrócitos, a hemoglobina liberada é transformada pelo nitrito de sódio em metaemoglobina que, associada com a azida de sódio, resulta na azidametaemoglobina que tem a absorção medida em dois comprimentos de onda, sendo a leitura da hemoglobina apresentada no visor digital do fotômetro, calibrado na fábrica de acordo com o método da cianometaemoglobina, que é o método de referência internacional para determinação da concentração de hemoglobina total do sangue.
Foram validadas as medidas da concentração de hemoglobina no sangue dos ratos utilizando o Coulter SPKS, no Laboratório da Fundação Hematologia e Hemoterapia (HEMOAM), cianometaemoglobina e hemoglobinômetro portátil HemoCue ${ }^{\circledast}$. Considerando a reprodutividade dos métodos, optou-se pela praticidade do HemoCue. O total de ferro ingerido pelos animais durante o período de repleção foi obtido multiplicando-se o consumo da ração pela concentração de ferro da ração. A quantificação do ferro incorporado à hemoglobina (ferro hemoglobínico) seguiu a metodologia preconizada por Whitaker et al. ${ }^{18}$. A biodisponibilidade de ferro determinada de acordo com o método de Mahoney et al. ${ }^{19}$ leva em consideração o ganho total de ferro hemoglobínico e o total de ferro ingerido pelos animais durante o período experimental. Os dados foram analisados utilizando-se o programa Microsoft Excel $97^{\circ}$ e a análise de variância para comparar as médias dos diversos parâmetros biológicos dos ratos foi complementada com o "Teste de Tukey - HSD" (Tukey Honest Significance Difference), considerando um nível de significância de $5 \%(p<0,05)$.

\section{RESULTADOSE DISCUSSÃO}

A concentração de ferro do açaí desidratado (Tabela 2) corrobora os achados de Yuyama et al. ${ }^{20}$. Entretanto, a literatura reporta valores superiores $(11,8 \mathrm{mg})$ para a concentração de ferro do açaí ${ }^{11}$, comprometendo a avaliação de ferro das dietas, em especial das populações para as quais o suco de açaí faz parte do hábito alimentar, o que sugere a necessidade de uma revisão mais criteriosa das tabelas de composição de alimentos. Embora não haja estudos comparáveis quanto à quantificação de fatores inibidores da absorção do ferro do açaí, os resultados encontrados para fibra alimentar e tanino não devem ser menosprezados (Tabela 2). As análises de composição centesimal das rações estão compatíveis com os cálculos realizados previamente na etapa de planejamento (Tabela 3). 
Tabela 2. Composição química do açaí e farinha de mandioca fortificada com ferro em $100 \mathrm{~g}$ da base seca.

\begin{tabular}{lcc}
\hline Composição & Açaí desidratado & Farinha de mandioca fortificada \\
\hline Umidade $(\mathrm{g} \%)$ & - & 8,00 \\
Proteína $(\mathrm{g} \%)$ & 10,30 & 1,42 \\
Lipídios $(\mathrm{g} \%)$ & 42,70 & 0,37 \\
Glicídios $(\mathrm{g} \%)$ & 34,10 & 89,45 \\
Cinza $(\mathrm{g} \%)$ & 0,44 & 0,73 \\
Fibra $(\mathrm{g} \%)$ & 12,50 & - \\
Ferro $(\mathrm{mg} \%)$ & 11,92 & 7,25 \\
Tanino $(\mu \mathrm{g} / \mathrm{g})$ & 232,00 & - \\
\hline
\end{tabular}

Tabela 3. Composição química das rações experimentais em $100 \mathrm{~g}$ da base seca.

\begin{tabular}{lrrrr}
\hline \multirow{2}{*}{ Composição } & \multicolumn{3}{c}{ Rações } \\
\cline { 2 - 5 } & \multicolumn{1}{c}{ AD } & \multicolumn{1}{c}{ FMF } & CA & AIN \\
\hline Umidade (g\%) & 2,51 & 6,16 & 2,36 & 7,74 \\
Proteína (g\%) & 19,31 & 18,35 & 17,42 & 18,43 \\
Lipídios (g\%) & 42,51 & 7,32 & 41,10 & 7,34 \\
Fração nifext (g\%) & 31,11 & 64,98 & 36,61 & 63,68 \\
Cinza (g\%) & 4,56 & 3,19 & 2,51 & 2,81 \\
Calorias (kcal) & 584,27 & 399,20 & 586,02 & 394,50 \\
Ferro (mg\%) & 4,59 & 5,61 & 4,45 & 4,93 \\
\hline
\end{tabular}

Rações: $A D=$ à base de açaí desidratado; $F M F=$ à base de farinha de mandioca fortificada; $C A=$ controle do açaí; AIN: American Institute of Nutrition = controle $($ AIN-1993 G)

Tabela 4. Peso, ganho de peso e consumo total de ração pelos ratos dos diferentes grupos.

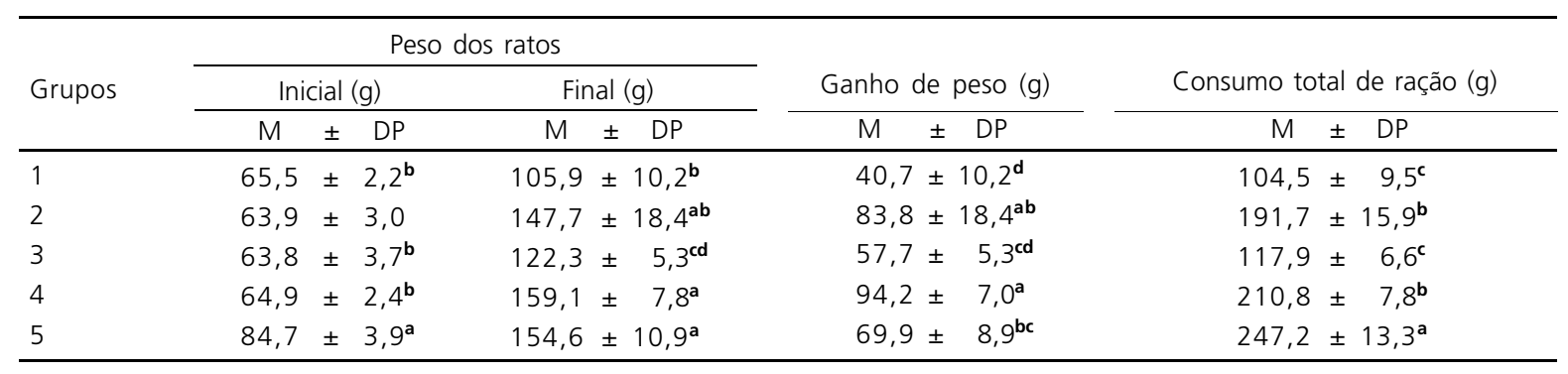

Grupos: 1 = ratos que receberam ração à base de açaí desidratado; 2 = ratos que receberam ração à base de farinha de mandioca fortificada; $3=$ ratos que receberam ração controle do açá; $4=$ ratos que receberam ração controle AIN -1993 G; $5=$ ratos que receberam ração comercial. As mesmas letras no sentido vertical não diferem em nível de $5 \%$ de probabilidade pelo teste de Tukey.

Ao final do período de depleção, a concentração média de hemoglobina dos animais que receberam a ração deficiente em ferro foi significativamente baixa quando comparada com a dos animais que recebem ração comercial (Tabela 4), demonstrando que o método utilizado foi adequado aos propósitos do estudo. Esses resultados são similares aos viabilizados em laboratório na presente pesquisa, utilizando a mesma metodologia ${ }^{22}$. Outros modelos por meio de sucessivos sangramentos do plexo orbital dos ratos são reportados na literatura ${ }^{23}$. 
As vantagens do modelo utilizado no presente estudo em relação aos demais métodos de depleção de ferro em ensaios biológicos são: maior rapidez de obtenção de ratos anêmicos com baixas concentrações de hemoglobina, utilização de menor número de animais, minimização do período de depleção, além de ser um método menos invasivo para os animais.

O peso médio inicial dos ratos anêmicos foi menor que o dos ratos normais, demonstrando que a deficiência de ferro tem influência não somente sobre a concentração de hemoglobina, mas também sobre o desenvolvimento dos animais na fase de lactação, refletindo um menor ganho ponderal. Ao final do período experimental, tanto os ratos que receberam o açaí como fonte de ferro como os ratos do grupo-controle do açaí apresentaram um menor consumo e ganho de peso (Tabela 4). Tais evidências podem ser justificadas pelo teor elevado de lipídio nas rações
(Tabela 3), o que pode ter comprometido o consumo de ração pelos ratos. $O$ açaí desidratado detém uma concentração de proteína na ordem de $10 \%$ e de glicídios na ordem de $34 \%$ (Tabela 2). Objetivando a manutenção das concentrações isoprotéica e isocalórica da ração à base de açaí, complementou-se com caseína e ajustou-se a sacarose. Apesar da mistura de fontes protéicas, açaí e caseína, acredita-se que a proporção utilizada tenha sido suficiente para a promoção do desenvolvimento dos ratos, inclusive para o grupo controle do açaí, cuja fonte protéica foi a caseína. As diferenças significativas encontradas nos parâmetros consumo de rações e ganho de peso foram corrigidas por meio dos cálculos de biodisponibilidade.

Entre os grupos experimentais houve recuperação de hemoglobina apenas nos animais que receberam a farinha de mandioca fortificada. A ração à base de açaí não foi eficaz na recuperação de hemoglobina ao final do período de

Tabela 5. Evolução da concentração média de hemoglobina dos diferentes grupos.

\begin{tabular}{|c|c|c|c|c|c|c|c|}
\hline \multirow{2}{*}{ Grupos } & \multicolumn{2}{|c|}{$\mathrm{Hb}$ inicial (g/dL) } & \multicolumn{2}{|c|}{$\mathrm{Hb}$ final $(g / d L)$} & \multicolumn{3}{|c|}{ Ganho de hemoglobina $(\mathrm{g} / \mathrm{dL})$} \\
\hline & $M \pm$ & DP & M & DP & $M$ & \pm & DP \\
\hline 1 & $4,8 \pm$ & $0,5^{b}$ & 5,2 & $\pm 0,7^{d}$ & 0,4 & \pm & $0,7^{c}$ \\
\hline 2 & $4,7 \pm$ & $0,5^{b}$ & 11,9 & $\pm 0,9^{b c}$ & 7,2 & \pm & $1,0^{a}$ \\
\hline 3 & $4,8 \pm$ & $0,5^{b}$ & 12,9 & $\pm 1,4^{\mathrm{ab}}$ & 8,2 & \pm & $1,7^{a}$ \\
\hline 4 & $4,7 \pm$ & $0,5^{\mathbf{b}}$ & 11,2 & $\pm 0,9^{c}$ & 6,4 & \pm & $1,1^{a b}$ \\
\hline 5 & $12,1 \pm$ & $0,4^{a}$ & 13,4 & $\pm 0,8^{a}$ & 1,2 & \pm & $0,7^{c}$ \\
\hline
\end{tabular}

Grupos: 1= ratos anêmicos que receberam ração à base de açaí desidratado; 2 = ratos anêmicos que receberam ração à base de farinha de mandioca fortificada; 3= ratos anêmicos que receberam ração controle do açaí; 4= ratos anêmicos que receberam ração controle AIN-1993 G; $5=$ ratos normais que receberam ração comercial; $\mathrm{Hb}=$ hemoglobina; as mesmas letras no sentido vertical não diferem em nível de $5 \%$ de probabilidade pelo teste de Tukey.

Tabela 6. Valores de ferro ingerido e ferro hemoglobínico dos ratos dos diferentes grupos e biodisponibilidade das diferentes rações.

\begin{tabular}{|c|c|c|c|}
\hline \multirow{2}{*}{ Grupos } & Ferro ingerido (mg) & Fero hemoglobínico (mg) & Biodisponibilidade (\%) \\
\hline & $M \pm D P$ & $M \pm D P$ & $M \pm D P$ \\
\hline 1 & $4,8 \pm 0,4^{c}$ & $0,5 \pm 0,2^{b}$ & $12,1 \pm 5,5^{c}$ \\
\hline 2 & $7,2 \pm 0,6^{\mathbf{b}}$ & $3,2 \pm 0,5^{a}$ & $44,6 \pm 3,6^{a}$ \\
\hline 3 & $5,8 \pm 0,3^{c}$ & $2,8 \pm 0,5^{a}$ & $48,9 \pm 7,6^{a}$ \\
\hline 5 & $9,4 \pm 0,3^{a}$ & $3,3 \pm 0,4^{a}$ & $34,9 \pm 4,2^{\mathbf{b}}$ \\
\hline 6 & - & $2,3 \pm 0,3^{a}$ & - \\
\hline
\end{tabular}

Grupos: 1= ratos que receberam ração à base de açaí desidratado; 2= ratos que receberam ração à base de farinha de mandioca fortificada; $3=$ ratos que receberam ração controle do açaí; 4= ratos que receberam ração controle AIN-1993 G; 5= ratos que receberam ração comercial. As mesmas letras no sentido vertical não diferem em nível de $5 \%$ de probabilidade pelo teste de Tukey. 
repleção, demonstrando a pouca expressividade do ferro (Tabela 5).

A baixa biodisponibilidade de ferro da ração à base de açaí (Tabela 6) pode ser justificada pela presença de inibidores da absorção de ferro, como tanino e fibra alimentar. A absorção do ferro não hemínico normalmente é baixa, cerca de $1 \%$ a $5 \%$, e varia substancialmente em função da presença de fatores químicos e dietéticos ${ }^{24}$. O tanino presente no chá e café é um inquestionável inibidor da absorção do ferro ${ }^{25}$. Estudos demonstram que uma xícara de café reduz a absorção de ferro de uma refeição à base de hambúrguer em 33\% e uma xícara de chá cerca de $64 \%{ }^{26}$. Desse modo, o tanino presente no açaí pode ter contribuído como fator antagonista da biodisponibilidade do ferro.

Por apresentar valores de atividade de água muito baixos, a farinha de mandioca pode ser considerada como produto seguro do ponto de vista microbiológico, o que reforça sua indicação como alimento veículo de ferro ou outro micronutriente.

No presente estudo, apesar da temperatura $\left(130^{\circ} \mathrm{C}\right)$ quando do processo de torrefação da farinha de mandioca, a retenção de ferro, oriundo do glicinato de ferro, foi de $94 \%$, indicando a viabilidade técnica da implantação de um processo de fortificação, utilizando-se tecnologia simples, no próprio local de produção da farinha.

A ração à base de farinha de mandioca fortificada demonstrou a eficácia da escolha do veículo de fortificação e do ferro de adição. Tais afirmações estão refletidas na concentração média e na recuperação de hemoglobina de ratos anêmicos que receberam a farinha (Tabela 5). Segundo Name \& Guerra ${ }^{9}$, entre os compostos de ferro conhecidos, o ferro aminoácido quelato é o que apresenta a maior biodisponibilidade em função de sua estrutura química, não interação com outros componentes da dieta, ausência de efeitos colaterais e alta aplicabilidade na tecnologia de alimentos. Estudo realizado na Guatemala demonstrou que $30 \mathrm{mg} /$ dia de ferro aminoácido quelato administrados por quatro semanas foi tão efetivo em aumentar a concentração de hemoglobina em adolescentes quanto $120 \mathrm{mg} /$ dia de sulfato ferroso ${ }^{27}$.

A variação da concentração de hemoglobina em ratos, na ordem de $11,4 \mathrm{~g} / \mathrm{dL}$ a $19,2 \mathrm{~g} / \mathrm{dL}$, está condicionada à raça, idade, sexo e estado de saúde do animal, sendo que para ratos machos Wistar com seis semanas, o valor preconizado é de $13,6 \mathrm{~g} / \mathrm{dL}^{28}$. De fato, a concentração de hemoglobina dos ratos do presente estudo que receberam ração comercial e farinha de mandioca fortificada com ferro corrobora os achados de Ringler \& Dabich ${ }^{28}$. Contudo, bons índices de hemoglobina e hematócrito podem ser alcançados com dieta isenta de suplementação ${ }^{29}$.

\section{O N CLUSÃ O}

Com base nos resultados apresentados, conclui-se que a concentração de ferro do açaí é baixa, de baixa biodisponibilidade e não recuperou a concentração de hemoglobina de ratos anêmicos. Sugere-se cautela ao recomendar a utilização do açaí como fonte de ferro.

O ferro da farinha de mandioca fortificada foi altamente biodisponível, o que permite a continuidade deste estudo, avaliando o impacto da introdução desse alimento na dieta de pré-escolares, grupo de risco para anemia.

\section{REFER Ê N CIAS}

1. Food and Agriculture Organization. World Health Organization. International conference on nutrition: Final report of the conference; 1992; Rome: FAONHO; 1992.

2. Garcia LYC, Mota ACA, Filho VO, Vaz FAC. Anemias carências na Infância. Pediatria. 1998; 20(2): 112-25.

3. Vannucchi H, Freitas LS, Szarfarc SC. Prevalência de anemias nutricionais no Brasil. Cad Nutr. 1992; 4:7-26.

4. Assis AMO. Estado da arte da anemia na adolescência: distribuição e implicações para a saúde. In: Publicação do Instituto Danone. Obesidade e anemia carencial na adolescência. São Paulo; 2001. p.33-46. 
5. Monteiro CA, Szarfarc SC, Mondini L. Tendência secular da anemia na infância na cidade de São Paulo (1984-1996). Rev Saúde Pública. 2000; 34(6):62-72.

6. Fisberg M, Naufel CCS, Braga JAP. National prevalence of anaemia in preschool Brazil: 10 capitals survey. Ann Nutr Metab. 2001; 45(Suppl 1):450.

7. Yuyama LKO, Nagahama D, Nozawa M, Souza CB, Alencar FH, Dantas HT, et al. Estado nutricional e ocorrência de anemia em lactentes atendidos na rede básica de saúde de Manaus-AM. In: Anais do 5 Congresso Nacional da Sociedade Brasileira de Alimentação e Nutrição; 1999; 3-5 dez. São Paulo: Sociedade Brasileira de Alimentação e Nutrição, 1999. p.154.

8. Agência Nacional de Vigilância Sanitária [Internet]. Brasília: ANVISA [atualizada 2002 dez 13; citado em 2003 abr 11]. RDC n 344. Disponível em: http:// www.anvisa.gov.br/legis/resol/2002/344_02 rdc.htm

9. Name JJ, Guerra JEF. Considerações sobre a biodisponibilidade do ferro como critério para o estabelecimento de recomendações de ingestão diária, com destaque para o ferro bis-glicina quelato. Food Ingredients. 2001; 12:3-14.

10. McKay RH, Higuchi DA, Winder WW, Feel RD, Brown $E B$. Tissue effects of iron deficiency in the rat. Biochem Biophys Acta. 1983; 757(3):352.

11. Reeves PG, Nielsen FH, Fahey GC Jr. AIN-93 - Purified diets for laboratory rodents: Final report of the American Institute of Nutrition Ad Hoc writing committee on the reformulation of the AIN-76A rodent diet. J Nutr. 1993; 123(11):1939-51.

12. Association of Official Analytical Chemists - Official Methods of Analysis. 12th ed. Manasha; 1995. $937 p$.

13. Asp NG, Johansson CG, Hallmer $H$, Siljestron $M$. Rapid enzymatic assay of insoluble and soluble dietary fiber. J Agric Food Chem. 1983; 31(3): 476-82.

14. Reicher F, Sierakowiski MR, Correa JBC. Determinação espectrofotométrica de taninos pelo reativo fosfotungstico-fosfomolibdico. Arq Biol Tecnol. 1981; 22(2):407-11.

15. Instituto Adolfo Lutz. Normas analíticas, métodos químicos e físicos para análises de alimentos. São Paulo; 1975.

16. Perkin-Elmer. Analytical methods for atomic absorption spectrophotometer. Norwalk; 1976.

17. Cornelis R. Use of references materials in trace element analysis of foodstuffs. Food Chem Barking. 1992; 43:307-13.
18. Whitaker P, Mahoney AW, Hendricks DG. Effect of iron deficiency anemia on percent blood volume in growing rats. J Nutr. 1984; 114(6):1137-42.

19. Mahoney AW, Van Orden CC, Hendricks DG. Efficiency of converting food iron into hemoglobin by the anemic rat. Nutr Metab. 1974; 17(4):223-30.

20. Yuyama LKO, Aguiar JPL, Silva Filho D, Yuyama K, Fávaro DIT, Vasconccellos MBA. Açaí como fonte de ferro: mito ou realidade? Acta Amazônica. 2002; 32(3):521-5.

21. Franco G. Tabela de composição química dos alimentos. 9.ed. São Paulo: Atheneu; 1999.

22. Silva SF, Aguiar JPL, Arakian SKL, Alencar FH, Yuyama LKO. Biodisponibilidade de ferro da dieta dos pré-escolares de diferentes ecossistemas da Amazônia. Estudo em ratos. In: Anais do 15을 Congresso Brasileiro de Nutrição, Segurança Alimentar e Nutricional no Brasil, Alimentação Saudável: um Direito Humano Universal; 1998, 22-6 ago. Brasília: Associação Brasileira de Nutrição; 1998. p.40.

23. Pedrosa ML, Rezende EG, Silva ME. Avaliação da biodisponibilidade de ferro da salsa (Petroselium sativam). In: Anais do 15으 Congresso Brasileiro de Nutrição, Segurança Alimentar e Nutricional no Brasil, Alimentação Saudável: um Direito Humano Universal; 1998, 22-6 ago. Brasília: Associação Brasileira de Nutrição; 1998. p.43.

24 Cook JD. Determinants of non-heme iron absorption in man. Food Tech. 1983; 37(10): 124-6.

25. Hallberg L. Bioavailable nutrient density: a new concept applied in the interpretation of food iron absorption data. Am J Clin Nutr. 1981; 34(10): 2242-7.

26. Morck TA, Lynch SR, Cook JD. Inhibition of food iron absorption by coffee. Am J Clin Nutr. 1983; 37:416-20.

27. Pineda O, Ashmead HD, Perez JM, Lemus C. Effectiveness of iron amino acid chelate on the treatment of iron deficiency in adolescents. J App Nutr. 1994; 46:2-11.

28. Ringler D, Dabich L. Hematology and clinical Biochemistry. In: The laboratory rat. San Diego: Academic Press; 1979.

29. Boaventura GT, Silva RHL, Tostes LF, Azeredo VB. Ganho de peso, hemoglobina e hematócrito de ratos recebendo dieta de Quissamã, RJ, com ou sem suplemento alimentar alternativo. Rev Nutr. 2003; 16(3):321-31.

Recebido para publicação em 4 de junho de 2003 e aceito em 31 de janeiro de 2005. 